\title{
On Internal Modeling of the Upright Postural Control in Elderly
}

\author{
H. Jafari ${ }^{1}$, M. Pauelsen ${ }^{2}$, U. Röijezon ${ }^{2}$, L. Nyberg ${ }^{2}$, G. Nikolakopoulos ${ }^{1}$ and T. Gustafsson ${ }^{1}$
}

\begin{abstract}
The second most common cause of injury in the elderly population is falling. In an effort to understand the mechanism behind the reduced ability to maintain balance in any posture or activity, we study the performance of the central nervous system as a controller of the body, while maintaining the balance in some postures or activities. Towards this direction, forty-five subjects aged over 70 were tested in different trials of quiet stance: a) hard stable surface with open eyes, b) stable surface with closed eyes, c) soft unstable surface with open eyes, and d) unstable surface, while eyes were closed. In the sequel, the body kinematics were described by legs and trunk segment angles in the sagittal plane, while the muscle activations were described by a weighted sum of rectified EMG signals from tibialis anterior and gastrocnemius muscles of left and right legs. Using the neuro-science hypothesis and adaptive control theory, a completely novel model was identified for the CNS based on the feedback internal model. The proposed model is able to predict the output commands, based on a recurrent neural network, while the efficiency of the proposed scheme has been proven based on multiple experimental results, showing that the model can sufficiently predict the muscle activity based on the optimum sensory inputs.
\end{abstract}

Keywords: posture, internal model, system identification, recurrent neural network, modeling

\section{INTRODUCTION}

Preserving the human body in a stable posture in upright stance can be a challenging task, since the human body is mechanically unstable [1]. The human brain, as a control system, is responsible to maintain the balance of the physical body in any posture or activity [2]. How the brain stabilizes the body, as well as the overall brain-body relation, are still fundamental open questions in the motor control field of study [3], thus studying the balance control is increasingly becoming a vital issue, since according to the World Health Organization (WHO), falls are the second dominant cause of injury and accidental death in older adults worldwide [4]. As the population of older adults is increasing throughout the world [4], the falls issue is an important problem that should be taken into proper consideration.

Towards this direction, the performance of the brain, namely the Central Nervous System (CNS), in the human balancing system, as well as its structure has been investigated for the last few decades. Preliminary, in [5] it has been identified that the CNS integrates different modalities of sensory information from visual, vestibular and proprioception.

\footnotetext{
*This work was funded by the Swedish Research Council Office under the Grant Agreement No.K2015-99X-22756-01-4

${ }^{1}$ Control Engineering Group, Department of Computer, Electrical and Space Engineering, Luleå University of Technology, Luleå SE-97187, Sweden

${ }^{2}$ Health and Rehabilitation Division of the Department of Health Sciences, Luleå University of Technology, Luleå SE-97187, Sweden.

Corresponding Author's email: (hedjafeltu.se)
}

Based on this sensorimotor integration, the CNS maintains the balance by sending proper motor command signals to the muscles, which create coordinated motor actions and reactions [6]. Aging or some age-related disabilities cause changes in important components of the postural control system, such as the musculoskeletal system and the sensory system, which decrease the balance abilities and are strongly related to higher risks of falls [7], [8]. How the CNS can adapt to the age-related changes and predict the appropriate motor commands to stabilize the body, has been a challenge for postural control research the latest years [3], [9].

The conceptual hypothesis of the CNS, which is called the Internal Model (IM) has received interest in the recent years with characteristic works to be in [9], [10], [11]. This theoretical model predicts the motor commands from the sensory inputs integration, during the changes in the environment and tries to address the issues of how the brain adapts to the body or to the environmental changes, as well as, how the brain predicts the motor commands with delayed and noisy sensory input signals. This model has been inspired by the first findings in [12], where it was studied the internal feedback in the cerebellum that can predict the movement and reduce the errors, while this feedback is called the efference copy, which is the copy of the motor command in the CNS.

However, this hypothetical representation of the CNS is identical to the adaptive control theory in the field of Automatic Control. An instructive review of the various adaptive control schemes, such as the Gain Scheduling [13], Adaptive Sliding Control [14] or Model Predictive Control [15], and their resembling biological IM is provided in [16]. In the last decades, with the achieved technological progress in computers and sensors, many researchers became more interested in collecting human data and trying to implement and validate the internal models by System Identification and Machine Learning techniques. Due to the cause and effect of the relationship of the input-output signals, these approaches can infer more practical models that can be validated based on further experimentation [17] and used in human-inspired robots like the one in [10]. For instance, in [18] the authors presented a system identification technique for the control strategies of humans in different situations, which was inspired by the IM hypothesis. Likewise, in [19] it was introduced a general IM model for motion learning by an Artificial Neural Network (ANN). Despite the flexibility of this approach to predict the motion, there was no consideration of the efference copy as a feedback to the system.

The contribution of this article focuses in the establishment and experimental validation of a novel model for the CNS 
in postural control of upright stance for elder adults, where the fall and maintaining balance is a concern. Within the framework of these criteria, the main contribution of the proposed model stems from: a) presenting a new predictive data driven model for the CNS in the upright stance, based on human experimental data, b) establishing of a novel adaptive representation of the CNS in upright stance by a Recurrent Neural Network (RNN), inspired by the IM hypothesis considering the efference copy as a feedback to the brain, and c) the ability to integrated multiple joints movements and degrees of freedom and adapt to the changes in the multiple sensory inputs. To the best of our knowledge, the proposed model is the first experimental verified model of the CNS in upright stance considering the adaptive behavior, while as it will be presented, it shows a higher capability in capturing the functionality of the CNS than the prevailing classical PID controllers.

\section{CONFIGURATION OF THE CNS MODEL}

The proposed schema of the conceptual CNS postural controller is presented in Fig. 1. To maintain the balance, the CNS receives feedback data, from multiple sensors in our body, such as vision, proprioception, and vestibular, with their corresponding delay time known as reaction time and as an output, generates motor commands to the muscles and skeletons of the body. In the proposed architecture, the joint angles signal $X$ and the rectified processed electromyography (EMG) signal $Y$ from muscles can be assumed as the input and the output of the model of CNS respectively.

To identify the dynamics of the CNS, from these received signals, three main steps should be followed. Initially, according to [20] and [21], the CNS restrain the multiple sensory variables and kinematics degrees-of-freedom to obtain the desired orientation. This procedure can be presented in the dimension reduction unit, while it should be noted that this reduction does not indicate elimination of the sensory signals, while on the other hand, it states how the CNS benefits, from all the received signals, to generate the flexible movement. In the proposed schema, the dimension reduction unit will be based on Principal Component Analysis (PCA) and will generate the uncorrelated reduced dimension signal $U$ as an input to the next steps.

In the recent perspective of the motor control [9], the cerebellum uses a copy of the motor command (efference copy) to predict the proper motor command and to correct the error between the desired movement and the actual one, while this regulation can happen in the adaptive module. Subsequently, the detected motor orientation signals $Z$ will be fed to the motor generation module to create the proper commands to the musculoskeletal system. In this scheme, these block units are implemented by the RNN, where the output of the reduction unit, with $z^{-d_{u}}$ time delay and feedback copy of the predicted multidimensional signal $\hat{Y}$ with a time delay $z^{-d_{y}}$, are stored as memory to predict the proper muscle activation signal. Finally, minimizing the error signal $E$ between the measured $Y$ and the predicted $\hat{Y}$

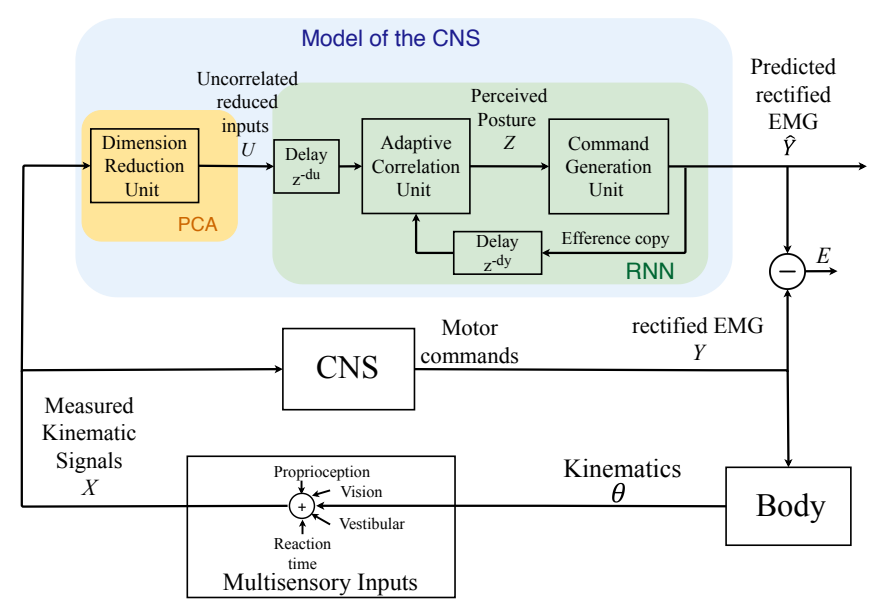

Fig. 1: Conceptual model for adaptive scheme of the CNS in human balance structure.

signal produced, by the proposed architecture, can be used to identify the characteristics and parameters of the system.

\section{A. PCA enabled dimension reduction Unit}

In the presented approach, the PCA will be utilized in order to transform the measured multidimensional kinematics signal $X$ from a set of correlated discrete time series data to a set of principal components $\Phi$, in such a way that all the components are orthogonal to each other and they derive the variance from the observation data set $x=$ $\left[x_{1}(k), x_{2}(k), \ldots, x_{p}(k)\right]$, with $p \in \mathbb{N}$ variables and $k \in \mathbb{N}$ sampling times [22], formally expressed as:

$$
\Phi_{p \times c}=X_{p \times k}^{T} A_{k \times c}
$$

where:

$$
\begin{aligned}
X_{p \times k}= & {[x(k), x(k+1), \ldots, x(k+n)] } \\
\Phi_{p \times c}= & {\left[\begin{array}{cccc}
\phi_{11} & \phi_{12} & \ldots & \phi_{1 c} \\
\phi_{21} & \phi_{22} & \ldots & \phi_{2 c} \\
\vdots & \vdots & \ddots & \vdots \\
\phi_{p 1} & \phi_{p 2} & \ldots & \phi_{p c}
\end{array}\right] }
\end{aligned}
$$

with $\Phi_{p \times c}$, with $p, c \in \mathbb{N}$, to be a square matrix of new components, where $c=p$ and $A_{k \times c}=$ $\left[a_{1}(k), a_{2}(k), \ldots, a_{c}(k)\right]$, and $k \in\{1,2, \ldots, N\}$ is the matrix of orthogonal eigenvectors of the variance-covariance matrix. For this formulation, there are many algorithms to calculate the $A$ matrix [23], while in the proposed approach the result is obtained by minimizing the error between the observation set and the uncorrelated observation set $X_{m}$ as:

$$
\left\|X-X_{m}\right\|_{2}
$$

The transported uncorrelated matrix $\Phi$ encloses the maximum possible variance of the observation data $X_{p \times k}$, such that the first component $\left(\phi_{11}, \phi_{21}, \cdots, \phi_{p 1}\right)$, is an axis in space with the largest variance, where the observations are projected to that axis. The rest of the components consist of perpendicular axes with descending variance. The number of components $c$ is the same as the number of variables of the 


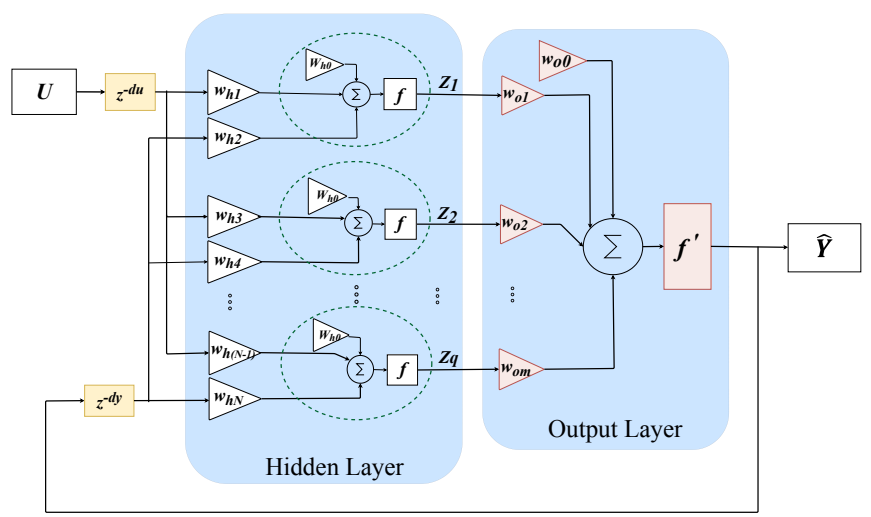

Fig. 2: Structure of NARX network in case of single hidden layer.

observation set $p$. However, consistently and especially in the case of correlated data, not all the components are necessary to be maintained as the first few components can represent a high percentage of the variance of the original data. Therefore, the components that present a small percentage of the variance can be removed.

Assuming $c^{\prime}<c, c^{\prime} \in \mathbb{N}$ components can represent more than $95 \%$ of the variance of the observation data, the new dimensioned reduced time series data, generated at this unit, may be formulated as:

$$
U=A_{k \times c^{\prime}}^{\prime}
$$

that will be routed to the RNN unit to generate and predict the muscle activation signals.

\section{B. Adaptive and motor generator unit}

Due to the efference copy as a feedback signal, the dynamics of this unit can be identified by an RNN. In this article, a Nonlinear AutoRegressive modeling approach, with eXogenous inputs (NARX) is utilized, since it can determine long time dependencies in predicting the time series signals that bypass the common problem of vanishing gradient in RNN [24].

The time series prediction scheme is formulated as:

$Y(k)=g\left(Y(k-1), \ldots, Y\left(k-d_{y}\right), U(k-1), \ldots, U\left(k-d_{u}\right)\right)$

where the function $g$ is approximated by a feed-forward neural network, with a time delay as an embedded memory [24], [25]. Figure 2 shows the structure of this network with one hidden layer and one output layer for multidimensional observation time series. More specifically, as an equivalent to the adaptive and motor generator unit, the network consists of two main components, which are the hidden and the output units. The hidden unit receives the output feedback with time delay and the outcomes of the reduction unit as exogenous inputs. In case of a single hidden layer, the outcome of each node, from this unit, may be written in the following form:

$$
\begin{gathered}
z_{q}(k)=f\left(w_{h_{0}}+\sum_{j=1}^{j=c \prime} \sum_{i=1}^{i=d_{u}} w_{h_{j}}^{i} u_{j}(k-i)+\right. \\
\left.\sum_{p=1}^{p=l} \sum_{m=1}^{m=d_{y}} w_{h_{p}}^{m} \hat{y}_{p}(k-m)\right)
\end{gathered}
$$

where $q \in\{1,2, \ldots, N\}$ is the number of nodes in the hidden unit, function $f$ is an activation function, $W_{h}$ are the weights of the hidden units, and the notations $\left(d_{u}, d_{y}\right)$ are the time delays of the exogenous inputs and feedback output respectfully. The outcome of the proposed RNN unit is denoted by $Z(k)=\left\{z_{1}, z_{2}, \ldots, z_{N}\right\}$ and it consists of the adapted perceived posture, which will be fed to the RNN unit to generate the motor commands and thus the outcome can be written in the following form:

$$
\hat{y}_{p}(k)=f^{\prime}\left(w_{o_{0}}+\sum_{q=1}^{q=N} w_{o_{q}} z_{q}(k)\right),
$$

where $f^{\prime}$ denotes the activation function and $W_{o}$ are the weights of the output units. By this configuration, the motor commands can be predicted by minimizing the error between the estimated value $\hat{y}(k)$ and the real measured value $y(k)$ by the following cost function:

$$
E(k)=\frac{\lambda}{l} \sum_{j=1}^{j=l}\left(\hat{y}_{j}(k)-y_{j}(k)\right)+\frac{(1-\lambda)}{n} \sum_{i=1}^{i=n} w_{i}^{2}(k)
$$

where $\lambda$ is the performance ratio and $W=\left\{w_{i} \mid \quad w_{i} \in\right.$ $\left.\left[W_{h}, W_{o}\right]\right\}$ are all the weights used in both hidden and output layer. The regularization term causes the network to be trained with smaller weights and biases, thus reducing overfitting to the actual data.

\section{EXPERIMENTAL METHODS}

The experiments were performed at the Human Health and Performance Lab - Movement Science at Luleå University of Technology, Luleå, Sweden. The study was executed in accordance with the Helsinki declaration and approved by the Regional Ethical Review Board in Umeå, Sweden (ref no. 2015-182-31). This study is part of a larger project [26], where participants were recruited from a community in Northern Sweden. Forty-five participants, 27 women and 18 men, were included in the analyses. Mean age was $75.2( \pm 4.5)$ years, mean height $167.2( \pm 9.9) m$, and mean weight $73.0( \pm 12.2) \mathrm{kg}$. An optic system with eight cameras for the 3D motion capture, the Qualisys Oqus 4 system was used together with a wireless system for EMG collaection, the Noraxon DTS 16 channel (Noraxon, USA). All data were sampled synchronically on a lab computer with Qualisys Track Manager (QTM) software, while a full body marker model with a total of 60 pieces of $10 \mathrm{~mm}$ round reflective markers was used for the motion capture measurements with a sampling rate of $200 \mathrm{~Hz}$. Four EMG sensors with Dual Electrodes 40x22mm (Noraxon, USA) were used to measure 
TABLE I: Measured signals of each subject.

\begin{tabular}{|c|c|c|c|}
\hline Inputs $(\mathrm{X})$ & Variable & Inputs $(\mathrm{X})$ & Variable \\
\hline Left ankle angle & $A n k_{L}$ & Right knee angle & Knee $_{R}$ \\
\hline Left knee angle & Knee $_{L}$ & Right hip angle & Hip $_{R}$ \\
\hline Left hip angle & $H i p_{L}$ & Pelvis-Thorax angle & Pel \\
\hline Right ankle angle & $A n k_{R}$ & Neck angle & Neck \\
\hline
\end{tabular}

\begin{tabular}{|l|c|}
\hline \multicolumn{1}{|c|}{ Outputs (Y) } & Variable \\
\hline EMG of tibialis anterior of left leg & $T A_{L}$ \\
\hline EMG of gastrocnemius of left leg & $G N_{L}$ \\
\hline EMG of tibialis anterior of right leg & $T A_{R}$ \\
\hline EMG of gastrocnemius of right leg & $G N_{R}$ \\
\hline
\end{tabular}

muscle activity of tibialis anterior and gastrocnemius bilaterally. Skin preparation and EMG electrode placement was in accordance with the instructions given by SENIAM [27] and with a sampling rate of $3000 \mathrm{~Hz}$. The postural behavior regarding the joint movements and the muscle activity was assessed during quiet stance in four different test scenarios of 30 seconds each: 1) stable, i.e. hard, surface with eyes open (SEO), 2) stable surface with eyes closed (SEC), 3) unstable, i.e. soft, surface with eyes open (UEO), and 4) unstable surface with eyes closed (UEC). To standardize foot placement, each test was performed with feet side by side and the first metatarsal heads at a distance equal to $75 \%$ of the width between the anterior superior iliac spines, with a self-chosen rotational angle of the foot placement. The test instructions were to stand up straight, look at the dot on the wall and stand as still as possible during the complete test. For the eyes closed trials, the participants were instructed to first look at the dot on the wall and then close their eyes. A trigger button was used to set a marker in the measurement to indicate initiation of the test when eyes were closed and the posture was stable. Table I summarizes the measured data of each subject during all the experimental scenarios.

\section{RESULTS}

The measured raw EMG signals and the measured kinematics angles were used for the system identification. Since sagittal plane movements are dominant in the quiet stance, the kinematics were restricted to the anterior-posterior directions.

\section{A. Data preprocessing and Statistical analysis}

Prior to using the EMG Signals to predict the muscle activation signals or the motor command from the brain, the raw signals are processed according to the standard recommendation by SENIAM [28]. According to the recommendation for surface EMG signal the motion artifacts and baseline noise should be removed by a high pass filter with a cut-off frequency between $(10-20 \mathrm{~Hz})$ [29]. Later the signal should be detrended, rectified and a low pass filter with a cut off frequency within the range of $(500-1000 \mathrm{~Hz})$ should be applied to obtain smoother signals [30].

After filtering and smoothing the input-output data for the CNS data-driven model, statistical tests were done to monitor
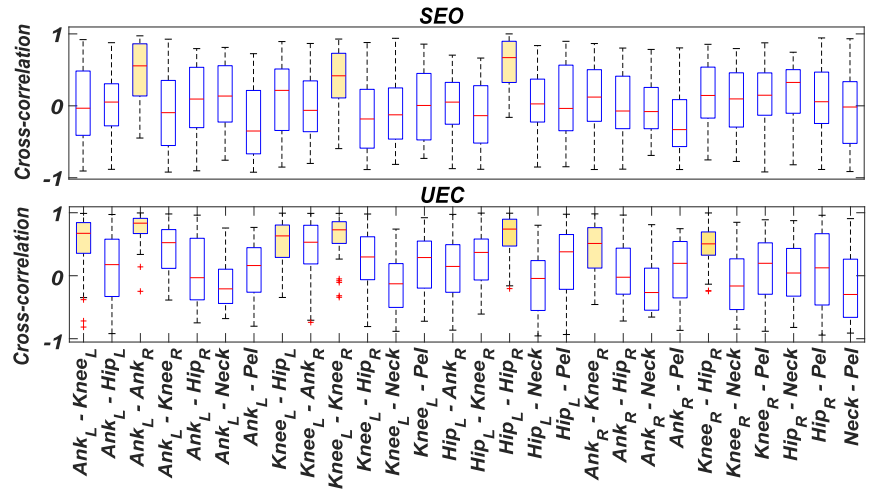

Fig. 3: Distribution of cross correlation of joints angles with zero lag in SEO and UEC scenario among 45 subjects. The yellow box plots indicate high correlation.

and analyze the important source of pattern and variation among the large data set. To observe the dependency of the 8 joints' angle to each other, cross-correlations were computed for each pair of joints of each subject through time.

In Figure 3 it is depicted the box plot of the crosscorrelation between each pair of the joints, with no time delay between them, during SEO and UEC trial among the population of the test. As it can be observed, for the majority of the subjects, there was a high correlation between the joints of the left and right legs $\left(A n k_{L}-A n k_{R}\right.$, Knee $_{L}-$ Knee $_{R}, \mathrm{Hip}_{L}-\mathrm{Hip}_{R}$ ). This is consistent with the statement in [1], where the human standing in quiet stance can be modeled by an inverted pendulum. On the other hand, the cross-correlation between other pairs of joints indicates that the movements are not just around the ankle for all the subjects, which is called "ankle strategy" and the model can be inverted to a multiple link inverted pendulum around different joints. Besides, it can be observed that in case of no vision and poor ankle proprioception, as in the case of the UEC test, the correlation between the movement of ankle and knee of the left leg was increased among the majority of subjects. The reason for this rather contradictory result is still not entirely clear, but it is probably related to the theory of UCM where in lack of sensory information and more challenging task, the brain tries to use the sensory data in a most favorable way and freezes the degrees of freedom to maintain the balance with the optimal strategy.

\section{B. Identification of the CNS}

Since it was observed that there is a correlation between the kinematic data, the reduction unit based on PCA was utilized to reduce the dimensions by considering the correlation between the data. Here, it was obtained that five components can be explained $95 \%$ of the variance of the input data, thus the 8 joints angle observation data can be reduced to five principal components for each subject. However, each individual has its unique distribution of input variables among the principal components. In fact, although PCA is a powerful tool to reduce the dimension of the system, which is more favorable in the RNN to avoid over-fitting, it cannot 
generalize an interpretation of each component. Since the goal of this article is to reduced the dimension and use the results for further analysis, the results are characterized as successful, while future work should concentrate on further analysis on each proposed components. To predict the rectified EMG signals from the reduction unit inputs by the NARX recurrent network, initially the filtered data are scaled and normalized so that all the inputs-outputs are in a comparable range and the global minimum of the cost function can be found faster. EMG signals are normalized based on the recommendation method of maximum voluntary contraction (MVC) value [27]. In the sequel, to validate the performance of the model identification, the data were separated to training (70\%) and testing datasets (30\%), which means 31 subjects were selected randomly for training the network and the rest of the subjects' data were kept to validate the model.

The network characteristics for the training, such as the number of the hidden layers (1layer), the number of nodes at each layer (5nodes) and the input delays $(150 \mathrm{msec})$, were selected to minimize the error of the network prediction, while the criteria to measure the performance and the error of prediction was the Root Mean Square Error (RMSE). To avoid over-fitting, the training data was also split to train and validation sets and for training the network, the Bayesian regularization back-propagation was selected. Figure 4 illustrates the 200 steps-ahead prediction of the normalized, rectified and filtered EMG signals in SEO test scenario. For better visualization, the results for three random subjects from the acquired test datasets are presented. As it can be observed, the model can predict the EMG signals up to 200 steps ahead $(1 \mathrm{sec})$ with a high accuracy $(R M S E \sim$ $0.055 \mu V)$. Remarkably, the model has the ability to adapt to each individual subject. This flexibility of the proposed model for the postural control to adapt to the changes of body characteristics of different subjects, makes it more preferable from the classical PID controllers.

The performance of the model for different steps ahead prediction is illustrated in Fig. 5. Expectedly, it can be seen that increasing the prediction time results in a bigger prediction error. However, due to the overall proposed methodology, it has been identified from the obtained results that the RMSE is kept small even in the case of the large predictions in time.

Moreover, to validate the performance of the CNS model to the changes of perceived multi sensory inputs, the model was trained with a training set from combination signal of SEC, UEO and UEC trials. Figure 6 presents the 200 step ahead prediction results for these scenarios on three random subjects. For better visualization only the results for the tibialis anterior muscle of left leg $T A_{L}$ are presented. The results show that the model is capable to adapt to the changes in the input signals and predicts the normalized and rectified EMGs with high accuracy (Average RMSE $=0.06 \mu \mathrm{V}$ for all subjects in the test data set).

\section{CONCLUSIONS}

By utilizing the neuro-science hypothesis and adaptive control theory, a completely novel model has been established for the CNS, based on an internal feedback model. As it has been indicated, the proposed model was able to predict the output commands, based on a recurrent neural network, while the efficiency of the proposed scheme has been demonstrated based on multiple experimental results. The overall scheme can adapt to physical body characteristics of different subjects, the changes of multiple sensory inputs and successfully predict the muscle activity based on the optimum number of sensory inputs.

\section{REFERENCES}

[1] D. A. Winter, A. E. Patla, F. Prince, M. Ishac, K. Gielo-Perczak, Stiffness control of balance in quiet standing, Journal of neurophysiology 80 (3) (1998) 1211-1221.

[2] R. Peterka, Sensorimotor integration in human postural control, Journal of neurophysiology 88 (3) (2002) 1097-1118.

[3] A. Karniel, Open questions in computational motor control, Journal of integrative neuroscience 10 (03) (2011) 385-411.

[4] W. H. Organization, Who global report on falls prevention in older age- fact sheet, http://www.who.int/mediacentre/ fact sheets/fs $344 /$ en/, Sidst set 30/01/2018 (2018 January).

[5] F. B. Horak, Clinical measurement of postural control in adults, Physical therapy 67 (12) (1987) 1881-1885.

[6] R. Johansson, M. Magnusson, M. Akesson, Identification of human postural dynamics, IEEE Transactions on Biomedical Engineering 35 (10) (1988) 858-869.

[7] R. Chiba, K. Takakusaki, J. Ota, A. Yozu, N. Haga, Human upright posture control models based on multisensory inputs; in fast and slow dynamics, Neuroscience research 104 (2016) 96-104.

[8] F. B. Horak, Postural orientation and equilibrium: what do we need to know about neural control of balance to prevent falls?, Age and ageing 35 (suppl_2) (2006) ii7-ii11.

[9] R. Shadmehr, M. A. Smith, J. W. Krakauer, Error correction, sensory prediction, and adaptation in motor control, Annual review of neuroscience 33 (2010) 89-108.

[10] I. V. Blagouchine, E. Moreau, Control of a speech robot via an optimum neural-network-based internal model with constraints, IEEE Transactions on Robotics 26 (1) (2010) 142-159.

[11] F. A. Mussa-Ivaldi, E. Bizzi, Motor learning through the combination of primitives, Philosophical Transactions of the Royal Society B: Biological Sciences 355 (1404) (2000) 1755-1769.

[12] D. Robinson, G. Lennerstrand, P. Bach-y Rita, Basic mechanisms of ocular motility and their clinical implications.

[13] A. D. Kuo, An optimal control model for analyzing human postural balance, IEEE transactions on biomedical engineering 42 (1) (1995) 87-101.

[14] J.-J. Slotine, J. Coetsee, Adaptive sliding controller synthesis for nonlinear systems, International Journal of Control 43 (6) (1986) 16311651.

[15] S. J. Sober, P. N. Sabes, Multisensory integration during motor planning, Journal of Neuroscience 23 (18) (2003) 6982-6992.

[16] C. Tin, C.-S. Poon, Internal models in sensorimotor integration: perspectives from adaptive control theory, Journal of Neural Engineering 2 (3) (2005) S147.

[17] L. Ljung, Perspectives on system identification, Annual Reviews in Control 34 (1) (2010) 1-12.

[18] X. Zhang, S. Wang, T. Seigler, J. B. Hoagg, A subsystem identification technique for modeling control strategies used by humans, in: American Control Conference (ACC), 2014, IEEE, 2014, pp. 2827-2832.

[19] J.-X. Xu, W. Wang, A general internal model approach for motion learning, IEEE Transactions on Systems, Man, and Cybernetics, Part B (Cybernetics) 38 (2) (2008) 477-487.

[20] M. L. Latash, J. P. Scholz, G. Schöner, Motor control strategies revealed in the structure of motor variability, Exercise and sport sciences reviews 30 (1) (2002) 26-31.

[21] M. L. Latash, M. F. Levin, J. P. Scholz, G. Schöner, Motor control theories and their applications, Medicina (Kaunas, Lithuania) 46 (6) (2010) 382. 

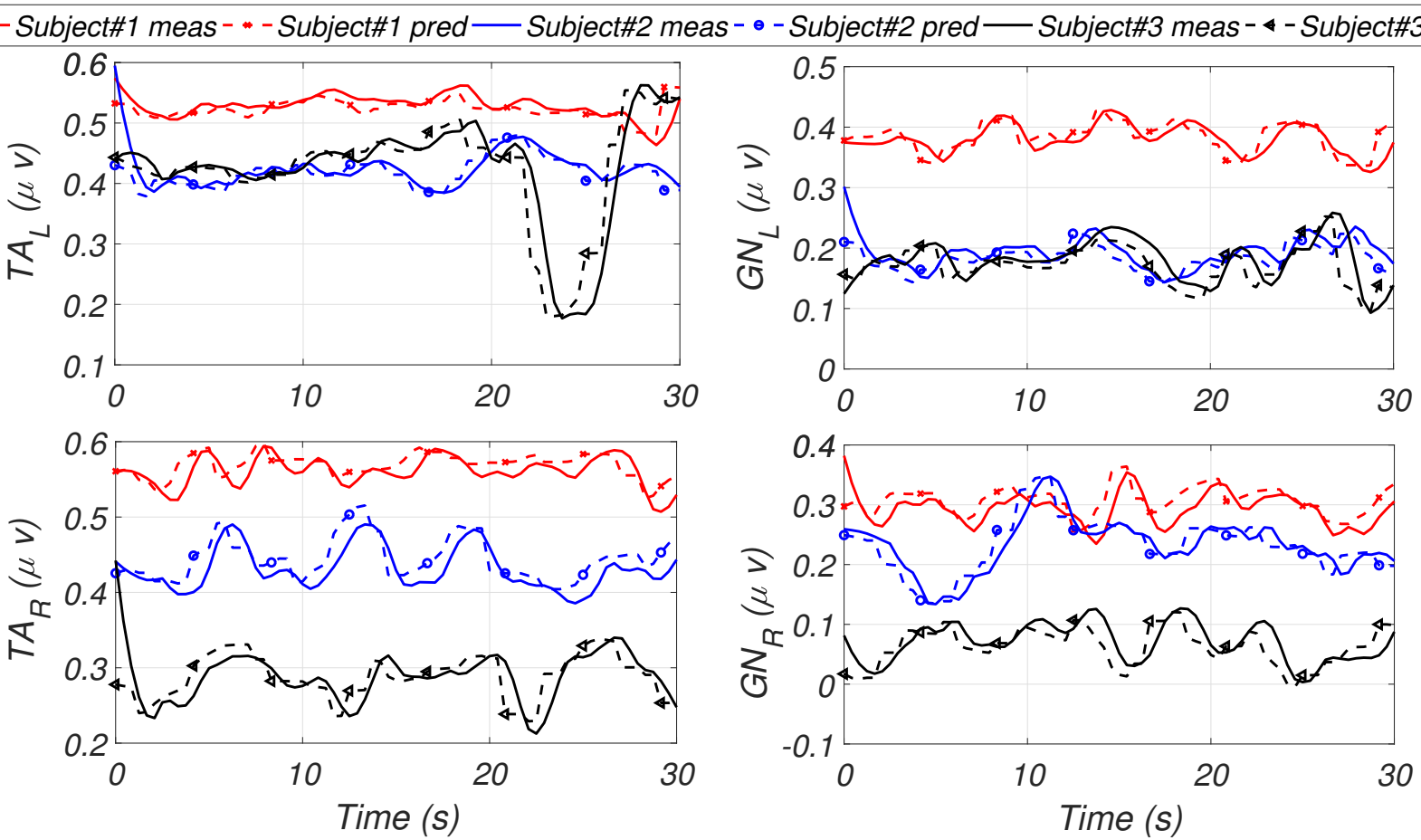

Fig. 4: Validation of 200 steps ahead prediction of normalized rectified and filtered EMG signals from four muscles in SEO test. The results are shown for three random subjects of the test data set. The measured EMGs and the predicted EMGs are presented by "meas" and "pred" respectively in the legend box.

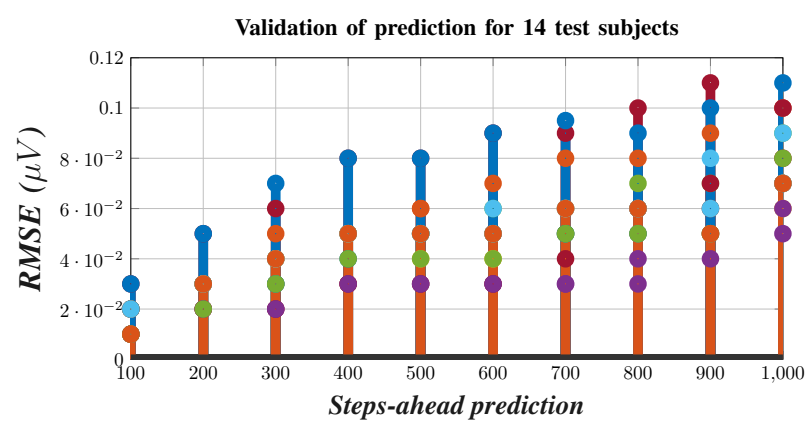

Fig. 5: Performance of the model for different steps a head prediction on the test data set. Each color bar indicates the prediction error for a subject during steps ahead prediction.

[22] S. Wold, K. Esbensen, P. Geladi, Principal component analysis, Chemometrics and intelligent laboratory systems 2 (1-3) (1987) 3752.

[23] A. A. Miranda, Y.-A. Le Borgne, G. Bontempi, New routes from minimal approximation error to principal components, Neural Processing Letters 27 (3) (2008) 197-207.

[24] E. Diaconescu, The use of narx neural networks to predict chaotic time series, Wseas Transactions on computer research 3 (3) (2008) 182-191.

[25] J. M. P. Menezes Jr, G. A. Barreto, Long-term time series prediction with the NARX network: An empirical evaluation, Neurocomputing 71 (16-18) (2008) 3335-3343.

[26] M. Pauelsen, L. Nyberg, U. Röijezon, I. Vikman, Both psychological factors and physical performance are associated with fall-related concerns, Aging clinical and experimental research (2017) 1-7.

[27] S. P. Group, Recommendations for sensor locations on individual muscles, http://seniam.org/, http://seniam.org/, Sidst set 30/01/2017 (2017 January).

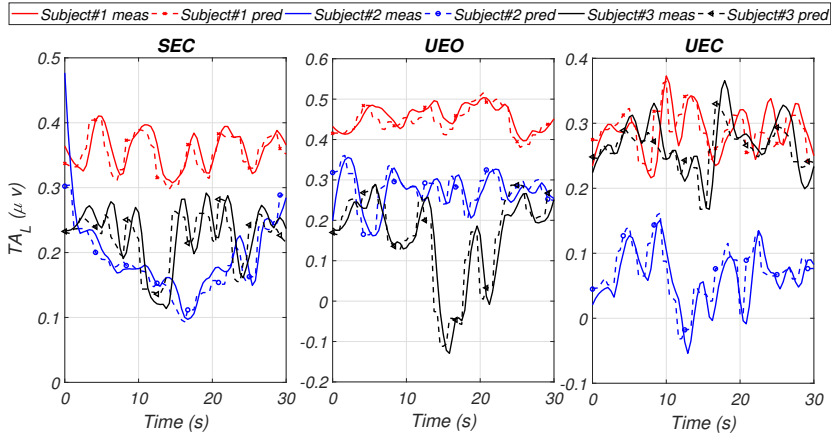

Fig. 6: Prediction results of tibialis anterior muscle of left leg by considering changes in multi-sensory inputs for three random subjects of the test data set. The measured EMGs and the predicted EMGs are presented by "meas" and "pred" respectively in the legend box.

[28] H. J. Hermens, B. Freriks, C. Disselhorst-Klug, G. Rau, Development of recommendations for semg sensors and sensor placement procedures, Journal of electromyography and Kinesiology 10 (5) (2000) 361-374.

[29] U. Mamikoglu, G. Andrikopoulos, G. Nikolakopoulos, U. Röijezon, M. Pauelsen, T. Gustafsson, Electromyography based joint angle estimation and control of a robotic leg, in: Biomedical Robotics and Biomechatronics (BioRob), 2016 6th IEEE International Conference on, IEEE, 2016, pp. 182-187.

[30] P. Konrad, The abc of emg, A practical introduction to kinesiological electromyography 1 (2005) 30-35. 